

\title{
Interactive/automated Method to Count Bacterial Colonies
}

\author{
Fernando C. Monteiro \\ Polytechnic Institute of Bragança, Portugal \\ João Ribeiro \\ Polytechnic Institute of Bragança, Portugal \\ Ramiro Martins \\ Polytechnic Institute of Bragança, Portugal
}

\begin{abstract}
The number of colonies in a culture is counted to calculate the concentration of bacteria in the original broth; however, manual counting can be tedious, time-consuming and imprecise. Automation of colony counting has been of increasing interest for many decades, and these methods have been shown to be more consistent than manual counting. Significant limitations of many algorithms used in automated systems are their inability to recognize overlapping colonies as distinct and to count colonies on the plate boundary. This study proposes an interactive semi-automated counting system and a fully automated counting system using image processing methods which overcomes these problems. The proposed systems are capable to reduce the manpower and time required for counting colonies while taking account colonies both around the central area and boundary areas of a Petri dish.
\end{abstract}

Keywords: Agar Medium, Colony Counter, Colony Forming Unit, Colony Segmentation, Escherichia Coli, Image Segmentation, Interactive Methods, Petri Dish

\section{INTRODUCTION}

The growth and maintenance of bacteria on agar plates (Petri dishes) has long been a common practice in microbiology. The Colony Forming Unit (CFU) assay is universally recognized as the gold standard method for measuring the effect of radiation on cell viability, environmental control, food and beverage safety assessment and clinical laboratory exams. A significant example is the monitoring or quality control of drinking water, where bacteria such as Escherichia Coli, Enterococcus, Cryptosporidium and faecal coliforms are the main indicator of microbiological water quality for human consumption ((EPA), 2006). The culturing process starts by inoculating the specimen to be examined on the agar, thus a solution of the specimen is spread over the agar surface. After inoculation, bacterial cultures are incubated to reproduce good conditions for pathogens bacteria growth. Figure 1 shows a Petri dish with several Escherichia Coli colonies.

\section{Figure 1: Petri dish with Escherichia Coli bacterial colonies}

The number of colonies in a culture is usually counted manually to calculate the concentration of bacteria based on the assumption that each colony has raised from one single bacterium (colony forming unit, CFU). However, this process is time-consuming (sometimes, the human who counts the colonies need to realize the procedure during many hours or even days), tedious (it is a monotonous procedure) and error prone (with the fatigue, the human being has more tendency to not do the right evaluation). The counting results obtained depend on the human conducting the count. This variability is one of the sources of error in the 
colony counting process that, along with methodological differences between different laboratories or even within a laboratory, can result in considerable fluctuations in results (Bewes, Suchowerska, \& Mckenzie, 2008). Due to this, for cultures with high density of colonies, manual counting mostly uses estimation methods, making an extrapolation from a small section of the Petri dish. Automating the detection, counting and analysis of CFU offers significant benefits to eliminate the risk of subjectivity, bias and human error, increasing speed and accuracy, and delivering unprecedented data archiving and retrieval capabilities.

Commercial products exist to facilitate accurate colony counting, ranging from manual counting aids (e.g., counting pens) to all-in-one platforms including image acquisition, processing, and analysis. However, fully automated counting systems also capable of batch processing multiple images at once can be prohibitively expensive for small labs and large facilities may necessitate multiple counting instruments posing a significant budgetary challenge to many laboratories (Putman \& Burton, 2005). Yet, with the development of digital cameras and document scanners alternatives to commercial products has been proposed showing that it is not necessary to use costly hardware and imaging system to collect easily the images of bacterial colonies.

Most automated counting systems perform adequately when the colonies are well spaced, large, circular in shape and with good contrast from the background. When these assumptions are violated, most automated colony analysis systems can rapidly lose reliability, accuracy and utility. These obstacles include the need to handle confluent growth or growth of colonies that touch or overlap other colonies; the identification of each colony as a unit in spite of differing shapes, sizes, textures, colours, light intensities; the exclusion of colonies around periphery of the plate reducing statistical accuracy.

To address the above problems, the goal of this study is to design and implement a cost-effective, softwarecentred system that accepts general digital camera images as its input, for detecting as well as enumerating bacterial colonies in a fully automatic manner. An interactive semi-automatic system is also proposed to overcome any error from fully automatic system. The proposed systems are capable to reduce the manpower and time required for counting colonies while producing correct colony counting.

\section{BACKGROUND}

In different fields of microbiology, immunology and cellular biology, counting colonies of cells growing on agar plates is routine. However, everyone who has already counted colonies knows that this is hard work which takes a lot of time. Many groups have thought about an improvement of the counting system and the cited publications certainly won't cover all attempts, but no method has achieved a widespread use at all.

The colony counting can occur using different approaches, direct or indirect methods. An indirect method is the traditional plate count method, often preferred because is cheap. Some technologies can be used for quantification the microbial growth, such as membrane filtration, ATP bioluminescence, direct epifluorescent filter microscopy, spiral plating and membrane laser scanning fluorescence cytometry (Uppal \& Goyal, 2012). It should not be forgotten that the automatic counter methodology will be only an asset, if provides an excellent correlation with the results that would be obtained by a specialist.

The development of automated counting methods should take into account potential sources of conflict: confluent growth, colonies that touch or overlap the surrounding, be able to identify and enumerating as being of a different group each colony according the shape, size, texture, colour or light intensity. In addition, such methods must be capable of rejecting common artefacts such as imperfections in the agar, dust and edges of Petri dishes. This tool must be designed to deliver a high degree of accuracy in the count, and it is required reliability and reproducibility.

The previously developed automated colony counting methods relied on various image processing/analysis techniques for automated detection and counting of colonies on agar media. (Corkidi, Diaz-Uribe, FolchMallol, \& Nieto-Sotelo, 1998) presented a method that counts bacterial colonies exploring the properties of the surface of microbial colonies. The colonies found in Petri dishes are illuminated so those properties are highlighted. A multilevel threshold algorithm is used to separate and count colonies. This technique does not count colonies on the dish boundary. (Marotz, Lübbert, \& Eisenbeiss, 2001) proposed a computer 
system to detect the microbial colonies in agar Petri dishes with a pre-processing stage composed by the detection of the counting region, image scaling and thresholding. The recognition system is composed by parameter calculation, application of fuzzy logic to determine the local maxima and a measure of goodness to select pixels as potential objects centres, which are used in the final counting. (Barber, et al., 2001) presented a technique for counting the number of cell colonies when subjected to a certain dose of radiation. The proposed method uses a modified Hough transform, and was designed in order to deal properly with merged or nebulae colonies, which had not been achieved by any other method until then. The results were compared to the counts made by four human experts, and they came to the conclusion that the performances are statistically identical. (Dahle, Kakar, Steen, \& Kaalhus, 2004) employed a flatbed scanner to count colonies in 12 Petri dishes at a time. After staining, the Petri dishes were put on the specially designed racks used to fix the dishes in the same position from experiment to experiment and decrease shading. (Zhang \& Chen, 2007) presented an automatic colony counter for bacterial colony enumeration without any human intervention. Although it has high accuracy in images with coloured colonies, it has problems with those with transparent media. (Niyazi, Niyazi, \& Belka, 2007) developed the Clono-Counter, which uses three parameters, namely grey level, maximum area of one colony, and grey level distribution within the colony, for colony counting. Users need to have some experience to find suitable parameters, but some guidelines are provided to speed up the process. (Clarke, et al., 2010) proposed a low-cost, high-throughput colony counting system consisting of colony counting software and a consumer-grade digital camera or document scanner. The software NICE (NIST's Integrated Colony Enumerator) reads standard image formats, and therefore may be used in conjunction with many imaging systems. (Brugger, et al., 2012) used a Bayes classifier that is applied to count the final number of bacterial colonies. This step is necessary as some of the colonies are concatenated to form larger groups. Geometric properties such as ratio between major and minor axis of the group are used to verify the number of colonies contained in the group. The colonies that touch the agar boundary were removed. The results were highly correlated with the ones obtained from manual counting. The OpenCFU program created by (Geissmann, 2013) provides control over the processing parameters and can be used to count cell colonies and other circular objects.

\section{AUTOMATIC COLONY COUNTER}

When human operators examine a bacteria colony image, they gradually identify objects from the image. First, the dish/plate region, which is the largest object in the image, is identified. Second, within the $\mathrm{dish} /$ plate region, one starts to identify colonies based on some criteria such as colour and shape. If colonies are clustered together, the operator will try to separate the clustered colonies based on their best visual judgment. Once all colonies are identified, the operator counts the total number of colonies.

This section introduces procedures used in the colony counting automatic system which includes segmenting dish region from background, identifying colonies from central and boundary areas, separating aggregated colonies, and reporting colony counts.

\section{Colony detection}

Segmentation distinguishes foreground objects from the background. In background extraction, the image is segmented in background and Petri dish region. The dish region is then divided in central area and boundary area.

\section{Petri dish segmentation}

Background intensity variation is an important issue in these cases, especially those with white colonies grown on clear medium where we need to put the dish on a darker surface to enhance the contrast. Otherwise, the colonies cannot be easily seen, even by trained eyes.

To divide images of Petri dishes into two parts, the images of Petri dishes need to be extracted from the background. The process started by filtering the greyscale image with the median filter in order to remove noise and then the magnitude of the gradient was used in a thresholding process to isolate the dish area. By 
filling the holes surrounded by the most significant connected components, the region inside the border can be retrieved, as shown in Figure 2(b). This image is used as a mask image to enable the extraction of the Petri dish image from the background. The result is shown in Figure 2(c).

Figure 2: (a) Greyscale image of Figure 1; (b) mask of dish area obtained after hole-filling; (c) image of Petri dish extracted from background obtained as a product of $(b)$ and $(a)$.

Petri dish area is then divided into two parts: the central area of the Petri dish and the boundary area.

\section{Central area detection}

The greyscale image in Figure 2(c) shows that the Petri dish boundary is darker than that the central area. Thresholding can thus be used for the partition of the central area and boundary area. Figure 3(a) shows the thresholding results of Figure 2(c) followed by morphological open filter in order to remove thin structures. To restore the removed colonies in the central area, holes surrounded by foreground pixels are filled, as shown in Figure 3(b). This mask is used to extract the central region from the Petri dish area as shown in Figure 3(c).

Figure 3: (a) Thresholding result of Figure 2(c); (b) mask of central area obtained after hole-filling; (c) image of central area obtained as product of $(b)$ and Figure 2(c).

\section{Boundary area detection}

With the locations of the central area, segmentation of the boundary area can be performed by subtracting the central area mask (Figure 2(b) from the Petri dish area mask (Figure 3(b)). The results are shown in Figure 4(a). This mask is used to extract the boundary region from the Petri dish area as shown in Figure 4(b).

Figure 4: (a) Boundary area mask; (b) boundary area obtained as a product of (a) and Figure 2(c)

\section{Colony extraction and counting}

\section{Colony detection in the central area}

The bottom-hat transform is employed to correct the effects of non-uniform illumination as shown in Figure 5(a). This transformation uses a structuring element to remove the object from the image. The difference operation then yields an image in which only the removed components remain. Segmentation of the colonies from the image by thresholding is then applied from which colonies in the central area are extracted as shown in Figure 5(b). To derive a suitable threshold value for segmentation, only pixels in the central area are considered for histogram statistics.

Figure 5: (a) Results of bottom-hat transformation of Figure 3(c); (b) thresholding results of (a).

\section{Colony detection in the boundary area}

Colonies that grow around the edge of dishes are difficult to count because the dish edge has usually similar colour as that of colonies. Thus, when colonies are separated by grey level thresholding, the dish rim and unevenly shaded portions of the edges tend to be selected as colonies as well. Some groups have solved this problem by excluding this area from image processing (Corkidi, Diaz-Uribe, Folch-Mallol, \& Nieto-Sotelo, 1998; Brugger, et al., 2012). However, since the colonies were distributed over the whole surface area of the plate, excluding colonies around periphery of the plate may reduce statistical. (Barber, et al., 2001) partially solved the edge problem by dividing the image with a blank image to decrease the contrast of the edge and using fish eye optics to acquire images. However, this solution requires images to coincide perfectly, and the image division is also computationally demanding. 
In this study, it was followed a similar method as the one proposed by (Chiang, Tseng, He, \& Li, 2015). To derive a suitable threshold value for segmentation, only pixels in the boundary area are considered for histogram statistics. The thresholding result of Figure 4(a) is shown in Figure 6(a), from which both the rim and colonies are extracted.

Figure 6: (a) Thresholding result of Figure 4(b); rim elimination results determined using length/width ratio of 2 .

As shown in Figure 6(a), the image of the rim is longer and narrower than that of the colonies; therefore, its approximate length and width circularly and radically along the centre of the dish are used for elimination. Several parameters are used to extract the rim: the mean area of central colonies, the ratio between length and width and the eccentricity of the objects.

The variance of the segment width is evaluated based on their morphology.

\section{Clustered colonies separation}

Ideally, an isolated foreground object from the segmentation step corresponds to one colony. However, such an object may correspond to more than one colony because several colonies may aggregate together to form a larger cluster. Hence, to obtain an accurate colony count, those clustered colonies need to be separated. During the classification process, a filter based on morphological processing is applied to classify the components as 'individual colony' or 'multiple colonies' as shown in Figure 7. In this process each component is assessed by a particle filter that takes into account relationships between variables such as area, perimeter, aspect ratio (eccentricity) and solidity in order to determine whether or not a region is likely to be a valid individual colony.

Figure 7: (a) Total number of objects detected in central and boundary areas of the Petri dish; (b) objects classified as 'individual colonies'; (c) objects classified as 'multiple colonies'.

The currently used assumption is that a single colony segment is approximately a round shape such that the ratio of its minor and major axis lengths is close to 1 . In other words, the greater deviation the axial ratio is from 1, the higher the possibility that the segment contains more than one colony. On the basis of this assumption, a cutoff value on the ratio of 0.7 was used in order to obtain the candidate segments for applying watershed algorithm. Solidity gives the proportion of the pixels in the convex hull that are also in the region where the convex hull specifies the smallest convex polygon that can contain the region. A solidity value lower than 0.95 indicates a clustered colony.

Individual colonies (Figure 7(b)) are accepted for counting whilst the objects identified as merged colonies (Figure 7(c)). On the basis of morphological features, these merged colonies are segmented using a variant of the watershed algorithm on their distance-map. In this process the intensity gradient image and the watershed algorithm is used to divide clustered colonies in the image as water flood in a topographical surface. To illustrate the concept, we demonstrate the application of watershed algorithm in Figure 8 over one of the merged colony of Figure 7(c).

Figure 8: (a) Enlarged example of clustering colony; (b) Clustered colonies separated in the watershed operation

After all colonies had been properly split and identified, the final step is to acquire the total number of viable colonies by adding up the number of the segments that have been identified as colonies.

\section{INTERACTIVE COLONY COUNTER}


For most biologists, a moderate average deviation from colony counter systems (lower than 20\%) will often be negligible compared to noise generated by other experimental factors (Geissmann, 2013). Rather than trying to provide a perfect similarity to human counts, methods based on digital image processing should focus on reducing the human labour involved.

Given that bacteria colony classification is indeed a complicated task even for well-trained human operators, a small effort to help correcting final colony enumeration is required from the user in this interactive colony counter system. This is done via an interactive process in which the user is asked to select the non-marked colonies resulting from a semi-automatic colony detection process.

In this semi-automatic process, the algorithm will identify the 'individual colonies', using the process described in previous section, counts them, and put a green colour over them, as shown in Figure 9(a).

Figure 9: (a) Result from semi-automatic colony counter; (b) colony classification by user intervention

The system allows the user to manually mark the non-coloured colonies by clicking the mouse over the colonies. The marks are identified with a yellow point in order to indicate to the user that the colony has already been counted, as shown in Figure 9(b). The number of user marks is then added to the number of individual colonies, yielding the total number of colonies in the Petri dish. This interactive method provides a fast colony counting less tedious than totally manual counting and with a higher accuracy than fully automated process.

\section{EXPERIMENTAL RESULTS AND DISCUSSION}

To evaluate the performance of the proposed methods, 26 images of Escherichia coli colonies in Petri dishes were selected for the experiments. The spread plate technique with agar plates was employed for the culturing of bacterial colonies.

Five images were collected with a 5 megapixel mobile phone camera and the other 21, available from (Chiang, Tseng, He, \& Li, 2015), were acquired with a special apparatus in order to accentuate the dish area and to provide adequate contrast between the colonies and the background. In this system the plate was illuminated from below by a LED panel light due to its uniform illumination and thin shape.

Figure 10.a shows Escherichia Coli colonies on a Petri dish obtained with a mobile phone and Figure 10.b shows images obtained with the system described in (Chiang, Tseng, $\mathrm{He}, \& \mathrm{Li}, 2015$ ).

Figure 10: Escherichia Coli colonies on a Petri dish. (a) images obtained with a 5 megapixels mobile phone; (b) images available from (Chiang, Tseng, He, \& Li, 2015), obtained with special apparatus.

A user-friendly graphical user interface (GUI) that implements the image processing methods described in the previous section has been developed as Figure 11 shows. This GUI contains a load button, to open the image file, two buttons to choose the counting mode, automatic and semi-automatic methods, a text box with the number of counted colonies and a close button to finish the application. When the users open the image file and choose the automatic counting mode, the program will automatically run without any setting.

\section{Figure 11: Graphical user interface created for the colony counting}

To evaluate the performance of the proposed methods, the 21 images from (Chiang, Tseng, He, \& Li, 2015) were counted by the fully automatic and the interactive methods and also manually by five biomedical engineering students. The range of the number of colonies counted manually was from 29 to 687 and the range of the number of colonies counted automatically was from 24 to 591 . The results obtained were compared with other automatic counting systems - NICE (Clarke, et al., 2010), Clono Counter (Niyazi, Niyazi, \& Belka, 2007) and Chiang et al. (ACBCIA) method (Chiang, Tseng, He, \& Li, 2015). The NICE and Clono Counter require the user to manually select the counting area or threshold prior to the automated process while ACBCIA is a fully automated system. 
Figure 12 summarizes the steps followed in the fully automated proposed method.

Figure 12: (a) Original image; (b) Petri dish mask; (c) detected Petri dish; (d) mask of central area; (e) detected central area; $(f)$ colonies detected in central area; $(g)$ mask of boundary area $(h)$ detected boundary area; $(i)$ thresholded boundary area; $(j)$ colonies detected in boundary area; $(k)$ colonies detected in Petri dish; (l) colonies after clustered separation.

The statistical results of precision, recall, F-measure and absolute percentage error (APE) are shown in Table 1. The evaluation results for NICE, Clono Counter and ACBCIA were obtained from (Chiang, Tseng, $\mathrm{He}, \& \mathrm{Li}, 2015)$.

Table 1: Statistical results of colony enumeration with different methods

\begin{tabular}{|l|l|l|}
\hline Fully automated method & Precision & 0.9877 \\
& Recall & 0.8462 \\
& F-measure & 0.9093 \\
& APE & $16.41 \%$ \\
\hline Interactive method & Precision & 0.9952 \\
& Recall & 0.9900 \\
& F-measure & 0.9921 \\
& APE & $0.79 \%$ \\
\hline ACBCIA & Precision & 0.96 \\
& Recall & 0.96 \\
& F-measure & 0.96 \\
& APE & $3.37 \%$ \\
\hline NICE & Precision & 0.96 \\
& Recall & 0.91 \\
& F-measure & 0.93 \\
& APE & $7.2 \%$ \\
\hline Clono Counter & Precision & 0.79 \\
& Recall & 0.95 \\
& F-measure & 0.85 \\
& APE & $24.94 \%$ \\
\hline
\end{tabular}

From Table 1, the value of precision of the fully automated proposed method is greater than the other three automatic methods. However, the number of false negatives reduces the value of recall. The causes for this include the intensity difference among colonies: the intensity values of smaller colonies are lower and closer to that of the background of the Petri dish, leading to their elimination when the image was threshold; concatenated colonies mistaken for noise: to remove the rim, components that displayed a ratio between major and minor axis length greater than 2 are eliminated. Although this method removed most of the rim from the image, concatenated colonies may still have been treated as portions of the rim and removed (see Figure 6), thereby decreasing the number of retrieved colonies. The performance of the system can be further improved by overcoming these problems.

The values of precision, recall and F-measure of the interactive proposed system are greater than the other methods. Furthermore, the absolute percentage error of the proposed method is $0.79 \%$ while the ones of ACBCIA, NICE and Clono Counter are $3.37 \%, 7.20 \%$ and $24.94 \%$, respectively.

\section{CONCLUSION}

The proposed machine vision based method is a robust yet effective method for bacterial counter. It has the ability to detect the dish plate regions, isolate colonies on the dish plate and further separate the clustered colonies for accurate counting of colonies. The fully automated counting system has a promising performance in terms of precision and is efficient as demonstrated by the experiments. The interactive 
method showed a higher performance than all the others methods in terms of precision, recall and absolute percentage error.

The proposed systems are capable to reduce the manpower and time required for counting colonies while taking account colonies both around the central area and boundary areas of a Petri dish.

More efforts need to be made at improving the performance of the bacteria colony classification and enumeration in future work. In particular, to detect and distinguish different species of bacteria not only for well-isolated colony, but also for clustered colonies in the dish/plate. The ultimate goal is to accurately classify bacterial colonies according to their strain types and produce the correct count for each class, which could greatly benefit clinical studies.

\section{REFERENCES}

(EPA), U. S. (2006). Water quality standards review and revision. Washington, DC.

Barber, P. R., Vojnovic, B., Kelly, J., Mayes, C. R., Boulton, P., Woodcock, M., \& Joiner, M. C. (2001). Automated counting of mammalian cell colonies. Physics in Medicine and Biology, 46(1), 63-76.

Bewes, J. M., Suchowerska, N., \& Mckenzie, D. R. (2008). Automated cell colony counting and analysis using the circular Hough image transform algorithm (CHiTA). Physics in Medicine and Biology, 53(21), 5991-6008.

Brugger, S. D., Baumberger, C., Jost, M., Jenni, W., Brugger, U., \& Mühlemann, K. (2012). Automated counting of bacterial colony forming units on agar plates. PLoS ONE, 7(3), e33695.

Chiang, P., Tseng, M., He, Z., \& Li, C. (2015). Automated counting of bacterial colonies by image analysis. Journal of Microbiological Methods, 108, 74-82.

Clarke, M. L., Burton, R. L., Hill, A. N., Litorja, M., Nahm, M. H., \& Hwang, J. (2010). Lowcost, high-throughput, automated counting of bacterial colonies. Cytometry A, 77(8), 790797.

Corkidi, G., Diaz-Uribe, R., Folch-Mallol, J. L., \& Nieto-Sotelo, J. (1998). COVASIAM: an image analysis method that allows detection of confluent microbial colonies and colonies of various sizes for automated counting. Applied and Environmental Microbiology, 64(4), 1400-1404.

Dahle, J., Kakar, M., Steen, H. B., \& Kaalhus, O. (2004). Automated counting of mammalian cell colonies by means of a flat bed scanner and image processing. Cytometry A, 60(2), $182-188$.

Geissmann, Q. (2013). OpenCFU, a new free and open-source software to count cell colonies and other circular objects. PLoS ONE, 8(2), e54072.

Marotz, J., Lübbert, C., \& Eisenbeiss, W. (2001). Effective object recognition for automated counting of colonies in petri dishes (automated colony counting). Computational Methods and Programs in Biomedicine, 66(2-3), 183-198.

Niyazi, M., Niyazi, I., \& Belka, C. (2007). Counting colonies of clonogenic assays by using densitometric software. Radiation Oncology, 2(1), 1-3.

Putman, N., \& Burton, M. H. (2005). Simplified method to automatically count bacterial colony forming unit. Journal of Immunological Methods, 302(1-2), 99-102.

Uppal, N., \& Goyal, R. (2012). Computational approach to count bacterial colonies. International journal of Advances in Engineering \& Technology, 4(2), 364-372.

Zhang, C., \& Chen, W.-B. (2007). An Effective and Robust Method for Automatic Bacterial Colony Enumeration. International Conference on Semantic Computing (pp. 581-588). Irvine, Canada: IEEE. 


\section{KEY TERMS AND DEFINITIONS}

Agar Media: A gelatinous material derived from algae, specifically used as a culture medium of bacteria and other cells for diagnostic or laboratory experiments purpose.

Bacterial Colony: A pile of multiple microorganisms originated from one mother cell and genetically identical.

Colony Forming Unit: A measure of viable cells in which a colony represents an aggregate of cells derived from a single progenitor cell.

Petri Dish: A shallow dish consisting of two round, overlapping halves that is used to grow microorganisms on solid culture medium.

Escherichia coli: A bacteria that normally inhabit the intestine of humans and animals. Some strains of this bacteria are capable of causing disease under certain conditions when the immune system is compromised or disease may result from an environmental exposure. 